\title{
ON THE NUMBER OF NORMAL SUBGROUPS OF AN UNCOUNTABLE GROUP
}

\author{
R. G. BURNS, JOHN LAWRENCE and FRANK OKOH
}

(Received 15 January 1985; revised 23 August 1985)

\begin{abstract}
In this paper two theorems are proved that give a partial answer to a question posed by G. Behrendt and $P$. Neumann. Firstly, the existence of a group of cardinality $\boldsymbol{N}_{1}$ with exactly $\boldsymbol{N}_{1}$ normal subgroups, yet having a subgroup of index 2 with $2^{\aleph_{1}}$ normal subgroups, is consistent with ZFC (the Zermelo-Fraenkel axioms for set theory together with the Axiom of Choice). Secondly, the statement "Every metabelian-by-finite group of cardinality $\aleph_{1}$ has $2^{\aleph_{1}}$ normal subgroups" is consistent with ZFC.
\end{abstract}

1980 Mathematics subject classification (Amer. Math. Soc.): primary 20 A 15; secondary 16 A 27.

\section{Introduction}

We are interested here in the following question.

QUESTION 1. Given any uncountable cardinal $\mathfrak{m}$, does there exist a group of cardinality $\mathfrak{m}$ with at most $\mathfrak{m}$ normal subgroups, having a finite-index subgroup with $2^{\mathrm{m}}$ normal subgroups?

The analogous question for the countable case was answered affirmatively by G. Behrendt and P. M. Neumann in their paper [1], which concludes with the remark that they were unable to generalize their method to the situation of uncountable cardinals. Here we prove two, somewhat complementary, consistency results, which at least eliminate some of the directions in which one might be tempted to proceed in trying to settle Question 1. Partly for ease of presentation we shall throughout take $m=\aleph_{1}$, the first uncountable cardinal, although our

(C) 1986 Australian Mathematical Society $0263-6115 / 86 \$ A 2.00+0.00$ 
results hold for certain other uncountable cardinals as well. Our first main result shows that Question 1 cannot have a negative answer within the system ZFC.

THEOREM 1. The existence of a group of cardinality $\boldsymbol{\aleph}_{1}$ with exactly $\boldsymbol{\aleph}_{1}$ normal subgroups, yet having a subgroup of index 2 with $2^{\aleph_{1}}$ normal subgroups, is consistent with ZFC (the Zermelo-Fraenkel axioms for set theory together with the Axiom of Choice ).

(In fact, our construction yields the consistency with ZFC of the existence of such a group having only $\boldsymbol{\aleph}_{0}$ normal subgroups.)

The countable example of Behrendt and Neumann in [1] is, at least in its simplest form, abelian-by-finite. Our second main result shows that an uncountable example with the property given in Question 1, if there is one, will certainly have a more complex structure.

THEOREM 2. The statement "Every metabelian-by-finite group of cardinality $\aleph_{1}$ has $2^{\aleph_{1}}$ normal subgroups" is consistent with ZFC.

The key to both theorems is the known consistency with ZFC of the statement that $2^{\aleph_{0}}=2^{\aleph_{1}}$ (which holds for certain uncountable cardinals other than $\aleph_{1}$ also; see [6, Section 19]). In fact in proving Theorem 1 we exploit the same properties of the quasi-cyclic group $\mathbf{Z}_{p^{\infty}}$ as do Neumann and Behrendt [1], namely that while it has only $\boldsymbol{\kappa}_{0}$ subgroups, it has $2^{\aleph_{0}}$ endomorphisms, so that its direct square $\mathbb{Z}_{p^{\infty}} \oplus \mathbb{Z}_{p^{\infty}}$ has $2^{\boldsymbol{\aleph}_{0}}$ subgroups (one for each $\eta \in$ End $\mathbb{Z}_{p^{\infty}}$, namely $S_{\eta}=$ $\left.\left\{(x, x \eta) \mid x \in \mathbb{Z}_{p^{\infty}}\right\}\right)$. Thus in seeking an answer to Question 1, it is natural to ask for a module of uncountable cardinality with the analogous property.

QUeSTION 2. Does there exist a module of cardinality $\boldsymbol{\aleph}_{1}$ with only $\boldsymbol{\aleph}_{1}$ submodules but with $2^{\aleph_{1}}$ endomorphisms? (Note that by [3, Theorem 1] the underlying ring of such a module cannot be (right) Noetherian of cardinality less than $\aleph_{1}$.)

In the course of proving Theorem 1 it emerges that Question 2 cannot have a negative answer. (However this consistency result of course gets us no further with Question 1.)

Proposition 1. An affirmative answer to Question 2 is consistent with ZFC. (In fact it is consistent with ZFC that there exist such a module with only $\boldsymbol{\aleph}_{0}$ submodules.) 
In connexion with Question 2 the following simple result may also be of interest.

Proposition 2. A module of cardinality $\aleph_{1}$ with fewer than $\aleph_{1}$ submodules has at most $2^{\aleph_{0}}$ endomorphisms (in fact at most $\boldsymbol{\aleph}_{1}$ endomorphisms if it has only finitely many submodules).

If Question 2 should have an affirmative answer (i.e. within the system ZFC) then with an eye to the original Question 1 , we might ask more specifically

QUESTION 3. Is there a module of cardinality $\boldsymbol{\aleph}_{1}$ over a countable group ring $\mathbf{Z}[G]$, with only $\aleph_{1}$ submodules but with $2^{\aleph_{1}}$ endomorphisms?

(Note that in view of [3, Theorem 3] one cannot hope for an abelian group $G$ in an affirmative answer to this question.)

We thank Juris Steprāns for some helpful remarks.

\section{Proofs of Theorem 1 and the propositions}

Proof of Theorem 1. Denote by $D$ the direct sum

$$
D=\bigoplus\left\{A_{\alpha} \mid \alpha \in \aleph_{1}\right\},
$$

where each $A_{\alpha} \cong \mathbb{Z}_{p^{\infty}}$ for some (single) fixed odd prime $p$, and where $\boldsymbol{\aleph}_{1}$ is to be thought of as the least ordinal of cardinality $\aleph_{1}$ (and an ordinal as the set of its predecessors). For each $\alpha \in \boldsymbol{N}_{1}$ present $A_{\alpha}$ additively as follows:

$$
A_{\alpha}=\left\langle a_{\alpha, 1}, a_{\alpha, 2}, \ldots \mid p a_{\alpha, 1}=0, p a_{\alpha, i+1}=a_{\alpha, i}, i=1,2, \ldots\right\rangle \text {. }
$$

Let $B$ denote the discrete alternating group of permutations of the index set $\boldsymbol{\aleph}_{1}$, i.e. the group of all even permutations of $\boldsymbol{N}_{1}$ fixing all but finitely many elements; then an action of $B$ on $D$ is defined in the obvious way by

$$
\left(a_{\alpha, i}\right) g=a_{\alpha g, i}, \quad \alpha \in \aleph_{1}, \quad i=1,2, \ldots, \quad g \in B,
$$

so that $B$ merely permutes the $A_{\alpha}$ as wholes. For each $i=1,2, \ldots$, write $L_{i}$ for the $i$ th "layer" of $D$, i.e. for the subgroup of $D$ generated by all $a_{\alpha, i}, \alpha \in \aleph_{1}$; thus $L_{i}$ is a direct sum of $\boldsymbol{N}_{1} p^{i}$-cycles. It is clear that each $L_{i}$ is $B$-invariant. Furthermore any $B$-invariant subgroup $S$ of $L_{i}$ not contained in $L_{i-1}$ (where $L_{0}$ is defined as the trivial subgroup) will contain all elements of the form $a_{\mu, i}-a_{\nu, i}$ $\mu, \nu \in \aleph_{1}, \mu \neq \nu$. To see this observe first that for some $\alpha \in \aleph_{1}, S$ must contain an element of the form $a_{\alpha, i}+x$, where $x \in L_{i}$ is a (finite) sum of multiples of elements $a_{\beta, j}$ with $\beta \neq \alpha, j \leqslant i$. By applying to this element any permutation 
from $B$ fixing all these $\beta$ and sending $\alpha$ to some $\gamma \in \boldsymbol{\aleph}_{1}$ different from $\alpha$, we see that also $a_{\gamma, i}+x \in S$, whence $a_{\alpha, i}-a_{\gamma, i} \in S$, and then the application of any permutation in $B$ sending $\alpha$ to $\mu, \gamma$ to $\nu$, yields finally $a_{\mu, i}-a_{\nu, i} \in S$.

Write $M_{i}$ for the subgroup of $L_{i}$ generated by all $a_{\mu, i}-a_{\nu, i}$; it is clear that $M_{i}$ has index $p^{i}$ in $L_{i}$, and also that $M_{i}>M_{j}$ for $i>j$. It follows that each non-trivial $B$-invariant subgroup $S$ of $D$ is the union of a finite or countably infinite chain $S_{1}<S_{2}<\cdots$ of non-trivial subgroups, where each $S_{i}\left(=S \cap L_{i}\right.$ ) is either $L_{i}$ or $M_{i}$ or $M_{i}+L_{j}$ for some $0<j<i$; moreover, if $S_{i}=M_{i}$ or $M_{i}+L_{j}$, then $S_{k}=M_{k}$ or $M_{k}+L_{j}$, respectively, for all subsequent $S_{k}$ in the chain $(k>i)$. Hence $D$ contains only $\aleph_{0} B$-invariant subgroups (whence, icidentally, since $B$ is simple, it follows easily that the semi-direct product $D B$, a "permutational wreath product", has only $\aleph_{0}$ normal subgroups).

Now each endomorphism $\theta$ of

$$
\mathbb{Z}_{p^{\infty}}=A=\left\langle a_{1}, a_{2}, \ldots \mid p a_{1}=0, p a_{i+1}=a_{i}, i=1,2, \ldots\right\rangle
$$

determines an endomorphism $\theta_{D}$ of $D$, defined by

$$
\theta_{D}: a_{\alpha, i} \rightarrow a_{i} \theta \phi_{\alpha}, \alpha \in \aleph_{1}, \quad i=1,2, \ldots,
$$

where $\phi_{\alpha}: A \rightarrow A_{\alpha}$ is the obvious isomorphism; thus $\theta_{D}$ maps the summands $A_{\alpha}$ of $D$ "uniformly", and is easily seen to commute with the action of $B$ on $D$. Hence the $\mathbb{Z}[B]$-module $D$ has at least $2^{\aleph_{0}}$ endomorphisms, since card(End $\mathbb{Z}_{p^{\infty}}$ ) $=2^{N_{0}}$. Imitating [1], we now observe that for each $\theta_{D} \in$ End $D$ the set $\left\{\left(x, x \theta_{D}\right) \mid x \in D\right\}$ forms a submodule of $D \oplus D$, and that distinct elements of End $D$ give rise in this way to distinct submodules of $D \oplus D$. Hence $D \oplus D$ has at least $2^{\aleph_{0}} B$-invariant subgroups. Continuing as in [1], we let $T$ be a 2-cycle whose generator $t$ acts on $D \oplus D$ according to the rule $(x, y) t=(x,-y)$. This action turns $D \oplus D$ into a $\mathbf{Z}[B \times T]$-module, which since $p$ is odd is easily seen to have only $\boldsymbol{\aleph}_{0}$ submodules. From this and from the simplicity of $B$ it follows readily that the semi-direct product $R=(D \oplus D)(B \times T)$ is a group of cardinality $\boldsymbol{\aleph}_{1}$ with $\boldsymbol{\aleph}_{0}$ normal subgroups, but with a subgroup of index 2 , namely $U=$ $(D \oplus D) B$, with $2^{\aleph_{0}}$ normal subgroups.

Finally, to obtain the full theorem we need (somewhat artificially) to boost the number of normal subgroups to $\aleph_{1}$. To this end let $K$ be a field of characteristic 2 and cardinality $\boldsymbol{N}_{1}$, write $P=\operatorname{PSL}(2, K)$, and consider the $\mathbf{Z}[P]$-module $K^{2} \oplus K^{2}$, where $P$ acts naturally on each $K^{2}=K \oplus K$. Then (cf. the conclusion of [1]) the semi-direct product $Q=\left(K^{2} \oplus K^{2}\right) P$ has precisely $\aleph_{1}$ normal subgroups, and by using the simplicity of $\operatorname{PSL}(2, K)$ and the fact that $p \neq 2$, it follows (see below) that $R \times Q$, of cardinality $\boldsymbol{\kappa}_{1}$, has exactly $\boldsymbol{\kappa}_{1}$ normal subgroups, yet has a subgroup of index 2 , namely $U \times Q$, with at least $2^{\aleph_{0}}$ normal subgroups. The theorem now follows from the aforementioned consistency with ZFC of the statement that $2^{\kappa_{0}}=2^{\kappa_{1}}$. 
To see that $R \times Q$ has exactly $\aleph_{1}$ normal subgroups, consider it as the semi-direct product $W G$ of $W=(D \oplus D) \oplus\left(K^{2} \oplus K^{2}\right)$ by $G=(B \times T) \times P$, where the actions of $B \times T$ on $D \oplus D$ and of $P$ on $K^{2} \oplus K^{2}$ are as defined above, and where $B \times T$ and $P$ act trivially on $K^{2} \oplus K^{2}$ and $D \oplus D$, respectively. For each $N \unlhd W G$, write $N_{W}$ for its projection on $W$ and $N_{G}$ for its projection on $G$. Then, as noted in the concluding paragraph of [3], $N_{W}$ is a normal subgroup of $W G$ (contained in $W$ ), $N_{G}$ is a normal subgroup of $G$, and $N$ is determined by the groups $N_{W}, N_{G}$, and $N \cap W$, together with the map $f_{N}$ : $N_{G} \rightarrow W / N \cap W$ defined by $f_{N}: g \mapsto w(N \cap W)$, where $g w \in N$. It is not difficult to see that $f_{N}$ is well-defined and in fact a homomorphism, so that $N_{G} / \operatorname{Ker} f_{N}$ is either trivial or a 2-cycle (since $P$ and $B$ are non-abelian simple, while $W$ is abelian). Hence, for each choice of $N_{G}$, there are at most $\boldsymbol{N}_{1}$ possibilities for $f_{N}$. Since there are exactly $\boldsymbol{\aleph}_{1}$ choices for $N_{W}$ and $N \cap W$ (as normal subgroups of $W G$ contained in $W$; here we are using the fact that $p$ is odd while char $K=2$ ), and since there are only finitely many choices for $N_{G}$, it follows that indeed $W G=R \times Q$ has exactly $\aleph_{1}$ normal subgroups $N$.

Proof of Proposition 1. Consider the $\mathbb{Z}[B \times P]$-module $D \oplus\left(K^{2} \oplus K^{2}\right)$, where the actions of $B$ on $D$ and of $P$ on $K^{2} \oplus K^{2}$ are as defined in the preceding proof, and where $B$ and $P$ act trivially on $K^{2} \oplus K^{2}$ and $D$, respectively. It is straightforward to see that since the module $D$ has only $\aleph_{0}$ submodules and at least $2^{\aleph_{0}}$ endomorphisms, while the module $K^{2} \oplus K^{2}$ has exactly $\boldsymbol{\aleph}_{1}$ submodules, and also since $p \neq 2$, the module $D \oplus\left(K^{2} \oplus K^{2}\right)$ has exactly $\aleph_{1}$ submodules and at least $2^{\kappa_{0}}$ endomorphisms. Proposition 1 now follows by invoking as before the consistency with ZFC of the statement that $2^{\aleph_{0}}=2^{\aleph_{1}}$.

Proof of Proposition 2 (cf. the conclusion of [1]). Let $M$ be a module of cardinality $\boldsymbol{N}_{1}$ with fewer thatn $\boldsymbol{\aleph}_{1}$ submodules. Then, as in the proof of the theorem, $M \oplus M$ has at least card(End $M$ ) submodules. Consider the automorphisms $a, b$ of $M \oplus M$ defined by

$$
(x, y) a=(x+y,-y), \quad(x, y) b=(y, x) .
$$

It is a matter of direct calculation to see that the subgroup $C$ of At:t $(M \oplus M)$ generated by $a$ and $b$ is finite (since $a^{2}=b^{2}=1$ and $a b$ has order at most 6). If $N$ is any $C$-invariant submodule of $M \oplus M$, and if $(x, y) \in N$, then also

$$
(x, y)+(x, y) a-(x, y) b a=(x, x) \in N,
$$

and then further

$$
(x, x)-(x, y)=(0, x-y) \in N \text {. }
$$


Hence each $C$-invariant submodule of $M \oplus M$ is determined by its intersection with the diagonal of $M \oplus M$, together with its intersection with either summand, so that $M \oplus M$ has fewer than $\aleph_{1} C$-invariant submodules (say $\mathfrak{f}$ ), since $M$ has fewer than $\boldsymbol{N}_{1}$ submodules. (Note that, moreover, if $M$ has only finitely many submodules, then will also be finite.) By the Theorem and Corollary 1.4 of [4], this implies that $M \oplus M$ can have no chain of more than (card $C$ ) $\times \mathfrak{f} \leqslant 12 \mathfrak{f}$ (not necessarily $C$-invariant) submodules. Hence each submodule of $M \oplus M$ is generated by at most $12 \notin$ elements, so that $M \oplus M$ can have at most $\kappa_{1}^{12 f}$ submodules.

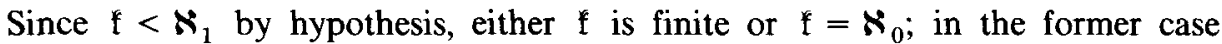
$\boldsymbol{N}_{1}^{12 \mathrm{f}}=\boldsymbol{N}_{1}$, and in the latter $2^{\boldsymbol{\kappa}_{0}}$. Since $M \oplus M$ has at least card(End $M$ ) submodules, the proposition follows.

\section{Proof of Theorem 2}

Theorem 2 follows readily (as shown below) from the following lemma (and from the known consistency with ZFC of the statement $2^{\kappa_{0}}=2^{\kappa_{1}}$ ). For the purpose of this lemma we assume that our rings all have a multiplicative identity, and that all modules are unital. A ring $T$ is said to be a finite normalizing extension of a subring $R$ if there exist elements $a_{1}, \ldots, a_{k} \in T$ (where we may suppose that $a_{1}=1$ ) such that $T=\sum_{j=1}^{k} a_{j} R$, and such that $a_{j} R=R a_{j}$ for $j=1,2, \ldots, k$.

LEMMA. Let $T$ be a finite normalizing extension of a commutative subring $R$ of cardinality $\leqslant \boldsymbol{N}_{0}$. Then every uncountable $T$-module $M=M_{T}$ contains at least $2^{\boldsymbol{N}_{0}}$ T-submodules.

(We note that the case $k=1$, i.e. $T=R$, of this lemma is just Theorem 4.2 of [7], and also that Theorem $C$ of [5] places strong limitations on the possibility of weakening the hypothesis that $T$ be a finite normalizing extension of $R$.)

To see how Theorem 2 follows from this lemma, suppose, as in that theorem, that $G$ is a group of cardinality $\aleph_{1}$ with a metabelian normal subgroup $H$ of finite index, and let $H^{\prime}$ denote the commutator subgroup of $H$. Then the integral group ring $\mathbb{Z}\left[G / H^{\prime}\right]$ is a finite normalizing extension of $\mathbb{Z}\left[H / H^{\prime}\right]$, and taking, in the notation of the lemma, $M=H^{\prime}, T=\mathbb{Z}\left[G / H^{\prime}\right], R=\mathbb{Z}\left[H / H^{\prime}\right]$ (with the appropriate actions on $\left.H^{\prime}\right)$, we infer from the lemma that if card $\left(H / H^{\prime}\right) \leqslant \boldsymbol{\aleph}_{0}$, then the $\mathbb{Z}\left[G / H^{\prime}\right]$-module $H^{\prime}$ has at least $2^{\aleph_{0}}$ submodules. In the group context this translates into the existence of at least $2^{\aleph_{0}}$ normal subgroups of $G$ contained in $H^{\prime}$, and then the desired conclusion follows from the consistency of the statement $2^{\aleph_{0}}=2^{\aleph_{1}}$ with ZFC. If, on the other hand, $\operatorname{card}\left(H / H^{\prime}\right)=\aleph_{1}$, then by 
[3, Theorem 1 and Note added in proof] $G / H^{\prime}$ has $2^{\aleph_{1}}$ normal subgroups, whence so does $G$.

Proof of Lemma. It is easily seen that it suffices to show that the module $\boldsymbol{M}=\boldsymbol{M}_{\boldsymbol{T}}$ contains a countably infinite "semi-independent" set $\left\{s_{1}, s_{2}, \ldots\right\}$ of elements, i.e. having the property that, for each $i=1,2, \ldots$, the element $s_{i}$ does not belong to the submodule of $M_{T}$ generated by all $s_{j} \neq s_{i}$.

This in turn will follow if we can show that $M_{T}$ must contain a "large" proper submodule, i.e. a module of cardinality $\geqslant \boldsymbol{\aleph}_{1}$. For, assuming this, we can define, in imitation of the proof of Lemma 4.1 of [7], a descending chain $M_{1} \supset M_{2} \supset \cdots$, of $T$-submodules of $M$, and a sequence of elements $s_{1}, s_{2}, \ldots$, such that, for all $n=1,2, \ldots$, we have

$$
\text { card } M_{n} \geqslant \aleph_{1}, \quad s_{n+1} \in M_{n}, s_{n+1} \notin s_{1} T+\cdots+s_{n} T+M_{n+1},
$$

and clearly a set $\left\{s_{1}, s_{2}, \ldots\right\}$ with these properties is semi-independent. To see how the presence in modules like $M_{T}$ of uncountable proper submodules allows the construction of such $M_{i}, s_{i}$, first set $M_{1}=M$ and choose $s_{1}$ to be any non-zero element of $M_{1}$. Suppose inductively that $M_{1} \supset \ldots \supset M_{k}$ and $s_{1}, \ldots, s_{k}$ have been defined (satisfying (1)). Writing $L=s_{1} T+\cdots+s_{k} T$, we clearly have card $L \leqslant \boldsymbol{\aleph}_{0}$, whence

$$
\operatorname{card}\left(\left(M_{k}+L\right) / L\right)=\operatorname{card} M_{k} \geqslant \boldsymbol{\kappa}_{1} .
$$

Hence, by our assumption, the $T$-module $\left(M_{k}+L\right) / L$ has a proper submodule of cardinality $\geqslant \boldsymbol{N}_{1}$. Let $H \subset M_{k}+L$ be the complete preimage of that module under the natural map $M_{k}+L \rightarrow\left(M_{k}+L\right) / L$. Then $H=\left(M_{k} \cap H\right)+L$, so that $M_{k} \cap H$ is a proper submodule of $M_{k}$ of cardinality $\geqslant \boldsymbol{\aleph}_{1}$. Define $M_{k+1}=$ $M_{k} \cap H$ and choose $s_{k+1} \in M_{k} \backslash H$ arbitrarily. It is then easily verified that $s_{k+1}$ and $M_{k+1}$ satisfy (1).

Thus we now have only to show that $M_{T}$ must contain a proper submodule of cardinality $\geqslant \aleph_{1}$. Assume the contrary. We may suppose without loss of generality that $M_{T}$ is faithful, since the property of being a finite normalizing extension is preserved under homomorphic images. Let $m \in M$ be any non-zero element of $M$ and let $B$ be a $T$-submodule which is maximal with respect to the property that $m$ and $B$ together generate the direct sum $m T \oplus B$. Then $m T \oplus B$ is "essential" in $M_{T}$, i.e. intersects non-trivially every non-zero $T$-submodule of $M$. By our assumption, $B$, and therefore also $m T \oplus B$, has cardinality $\leqslant \aleph_{0}$. Now in a similar manner take $C$ to be an $R$-submodule of $M_{R}$ such that $[(m T \oplus B) \oplus C]_{R}$ is essential in $M_{R}$. We claim that $m T \oplus B \oplus C \neq M$. To see this, suppose the contrary, and consider the map

$$
\psi: C \rightarrow(m T \oplus B)^{k}, \quad c \mapsto\left(c a_{1} \downarrow, \ldots, c a_{k} \downarrow\right),
$$


from $C$ to the Cartesian product of $k$ copies of $m T \oplus B$, where, as above, $T=\sum_{i=1}^{k} a_{i} R$, and where $c a_{i} \downarrow$ denotes the projection of $c a_{i}(\in M=m T \oplus B \oplus C$ by assumption) onto $m T \oplus B$. Since $\operatorname{card}(m T \oplus B) \leqslant \boldsymbol{\aleph}_{0}$ and card $M>\boldsymbol{\aleph}_{0}$, so that we must have card $C>\boldsymbol{\aleph}_{0}$ for $M=m T \oplus B \oplus C$ to hold, there must certainly be two distinct elements $c_{1}, c_{2}$ of $C$ such that $\psi\left(c_{1}\right)=\psi\left(c_{2}\right)$. However, since $\psi\left(c_{1}-c_{2}\right)=\psi\left(c_{1}\right)-\psi\left(c_{2}\right)$, we then have $\psi\left(c_{1}-c_{2}\right)=0$, whence $\left(c_{1}-\right.$ $\left.c_{2}\right) a_{i} \in C$ for $i=1, \ldots, k$. It follows from this that the $T$-submodule $\left(c_{1}-c_{2}\right) T$ is contained in $C$, which contradicts the essentiality of the $T$-submodule $m T \oplus B$. Hence the essential $R$-submodule $[m T \oplus B \oplus C]_{R}$ must be proper in $M_{R}$, as claimed.

By [2, Lemma 1.2] the largest $T$-submodule $N_{T}=N$ contained in $m T \oplus B \oplus C$ has the property that $N_{R}$ is essential in $M_{R}$. As $N_{T}$ is a proper submodule of $M_{T}$, we must have card $N \leqslant \aleph_{0}$ by assumption. For each $a \in M$, define the ideal $E(a)$ to be $\{r \in R \mid a r \in N\}$, and then, for each $a \in M, j=1, \ldots, k$, define the ideal $E(a, j)$ by

$$
E(a, j)=\left\{r \in R \mid \exists s \in E(a) \text { such that } s a_{j}=a_{j} r\right\} .
$$

It follows from the essentiality of $N_{R}$ in $M_{R}$ that each $E(a)$ is an essential ideal of $R$ (i.e. intersects non-trivially every non-zero $R$-ideal), and thence that the $E(a, j)$, and therefore also the intersections $E^{*}(a)=\bigcap_{j=1}^{k} E(a, j)$, are all essential $R$-ideals. The ideals $E^{*}(a)$ have the further property that, for each $r \in E^{*}(a)$, and for each $j=1, \ldots, k$, there is an element $s_{j} \in E(a)$ such that $s_{j} a_{j}=a_{j} r$. We now define a map $\gamma: M \rightarrow R^{k}$ (where $R^{k}$ denotes the Cartesian product of $k$ copies of $R$ ) as follows: for each $a \in M$ choose a nonzero element $b \in E^{*}(a)$, then choose $b_{1}, b_{2}, \ldots, b_{k} \in E(a)$ such that $b_{j} a_{j}=a_{j} b$, and then set $\gamma(a)=$ $\left(b_{1}, \ldots, b_{k}\right)$. (Note that since $a_{1}=1$, we have $b_{1}=b$.) Since card $R \leqslant \boldsymbol{\aleph}_{0}$, and since $M$ is uncountable, there is an uncountable subset $S_{0}$ of $M$ such that $\gamma\left(s_{1}\right)=\gamma\left(s_{2}\right)$ for all $s_{1}, s_{2} \in S_{0}$. Define a map $\delta: S_{0} \rightarrow N^{k}$ by

$$
s \mapsto\left(s b_{1}, \ldots, s b_{k}\right) \text {, where }\left(b_{1}, \ldots, b_{k}\right)=\gamma(s) \text {. }
$$

Once again, since $n$ is countable while $S_{0}$ is not, there must be an uncountable subset $S$ of $S_{0}$ such that $\delta\left(s_{1}\right)=\delta\left(s_{2}\right)$ for all $s_{1}, s_{2} \in S$. Hence the $T$-submodule $K$ of $M$ generated by the set $\{x-y \mid x, y \in S\}$ will also be uncountable. We now complete the proof by showing that $K$ is in fact a proper submodule. This we do by finding a non-zero element $r$ of $R$ which annihilates it; it will then follow that $K \neq M$, since $M$ is faithful by assumption. For all $s \in S, \gamma(s)$ is constant, say $\gamma(s)=\left(b_{1}, \ldots, b_{k}\right)$. Take $r=b_{1}$. Then for any $x, y \in S$, and for any element $\sum_{j=1}^{k} a_{j} r_{j} \in T$, we have

$$
(x-y)\left(\sum_{j=1}^{k} a_{j} r_{j}\right) b_{1}=\sum_{j=1}^{k}(x-y) a_{j} b_{1} r_{j}=\sum_{j=1}^{k}(x-y) b_{j} a_{j} r_{j}=0,
$$

where the last equality follows from the fact that $\delta(x)=\delta(y)$. 


\section{References}

[1] Gerhard Behrendt and Peter M. Neuman, 'On the number of normal subgroups of an infinite group', J. London Math. Soc. (2) 23 (1981), 429-432.

[2] J. Bit-David and J. C. Robson, 'Normalizing extensions I' (Ring Theory, Antwerp 1980, Lecture Notes in Mathematics, Vol. 825, Springer-Verlag, New York, 1980).

[3] R. G. Burns, Frank Okoh, Howard Smith, and James Wiegold, 'On the number of normal subgroups of an uncountable soluble group', Arch. Math. 42 (1984), 289-295.

[4] J. W. Fisher, 'Finiteness conditions for modular lattices with finite group action', Canad. $J$. Math. 31 (1979), 558-564.

[5] B. Hartley, 'Uncountable Artinian modules and uncountable soluble groups satisfying Min - $n$ ', Proc. London Math. Soc. (3) 35 (1977), 55-75.

[6] Thomas Jech, Set Theory (Academic Press, New York, San Francisco, London, 1978).

[7] J. Steprāns, 'The number of submodules', Proc. London Math. Soc. (3) 49 (1984), 183-192.

Department of Mathematics

York University

North York, Ontario

Canada M3J 1P3

Department of Mathematics

Wayne State University

Detroit, Michigan 48202

U.S.A.
Pure Mathematics

University of Waterloo

Waterloo, Ontario

Canada N2L 3G1 